\title{
Review of HPLSE special issue on target fabrication
}

\author{
Christopher Spindloe ${ }^{1,2}$, Yuji Fukuda ${ }^{3}$, Paul Fitzsimmons ${ }^{4}$, Kai Du ${ }^{5}$, and Colin Danson ${ }^{6,7}$ \\ ${ }^{1}$ Science and Technology Facilities Council, Rutherford Appleton Laboratory, UK \\ ${ }^{2}$ Scitech Precision Ltd, Rutherford Appleton Laboratory, UK \\ ${ }^{3}$ Kansai Photon Science Institute, QST, Kyoto, Japan \\ ${ }^{4}$ General Atomics, San Diego, USA \\ ${ }^{5}$ Research Center of Laser Fusion, CAEP, Mianyang, China \\ ${ }^{6}$ AWE plc, Aldermaston, $U K$ \\ ${ }^{7}$ CIFS, Blackett Laboratory, Imperial College London, UK \\ (Received 24 February 2018; accepted 1 March 2018)
}

Keywords: high-power laser; high-power laser-related laser components; target design and fabrication

In 2017 the journal High Power Laser Science and Engineering produced a Special Issue on Target Fabrication. The scope of the special issue was to span the latest developments and reviews on topics related to their deployment on ultrahigh-energy/power laser facilities. The topics invited for inclusion were:

- Target assembly

- Novel characterization techniques

- Target solutions for high repetition rate targets

- Cryogenic target development

- Novel material fabrication

- Precision micro-machining

- Target debris studies

Although this was not meant as an exhaustive list. As is usual with a Special Issue of this type Guest Editors were invited to lead in sourcing articles. These editors were: Paul Fitzsimmons, General Atomics, USA; Chris Spindloe, STFC Rutherford Appleton Laboratory, UK; Professor Yuji Fukuda, Kansai Photon Science Institute, Japan; and Professor Kai Du, China Academy of Engineering Physics, China.

The field of target fabrication is very diverse, often being led by the fundamental research priorities of the

Correspondence to: C. Spindloe, Science and Technology Facilities Council, Scitech Precision Ltd, Rutherford Appleton Laboratory, Harwell Campus, Oxon, UK. Email: christopher.spindloe@stfc.ac.uk national laboratories across the world. Submissions for the special issue covered the invited topics and also branched out into broader areas and capabilities. Papers described research on: techniques for the production of targets for inertial confinement fusion (ICF) ${ }^{[1-8]}$, micro-assembly ${ }^{[3,8,9]}$, liquid targets ${ }^{[10]}$, systems for delivery of targets at high repetition rates ${ }^{[5,11,12]}$, fundamental target fabrication processes ${ }^{[2,4,13-16]}$, low density materials $^{[15,17,18]}$ and metrology methods ${ }^{[7,18,19]}$. In addition, there were three important review papers ${ }^{[1,5,12]}$ that give an excellent snapshot of the current capabilities across the world.

\section{Review papers}

There are three review papers. A comprehensive review paper $^{[12]}$, contributed to by 47 authors from 26 institutions across 10 countries, is included on the requirements for high repetition rate targets. A review paper summarizing the development of capabilities in target fabrication for ICF research in China at the Research Centre of Laser Fusion in Mianyang is also included ${ }^{[1]}$ and a paper summarizing the current status of layering techniques and target development for laser inertial fusion energy around the world is authored by the Lebedev and Moscow Engineering Physics Institute $^{[5]}$.

\section{Inertial confinement fusion}

A range of papers that cover the design of the targets from a review of the developments in China ${ }^{[1]}$ to recent work in the United States including the manufacture of 
$30 \mu \mathrm{m}$ diameter fill tubes ${ }^{[2]}$ and subsequent ice layering. The assembly of targets using precision multi-axis manipulators ${ }^{[3,8]}$; and the relevance of this assembly process to other target micro-assembly processes are published. Papers on ablator materials including different beryllium copper/carbon compound mixes ${ }^{[4]}$ and the fundamental physical properties of the films and coatings were published. Characterization of shells using atomic force microscope (AFM) techniques ${ }^{[7]}$ and hohlraum manufacture and leak characterization papers $^{[6]}$ were published. Cryogenic filling and layering of shells are addressed in a wide ranging paper ${ }^{[5]}$.

\section{Micro-assembly}

Assembly techniques for the manufacture of complex assemblies for ICF experiments on the National Ignition Facility (NIF) ${ }^{[8]}$ and ShenGuang III ${ }^{[3]}$ were presented with emphasis on robotic assembly as a route to increase accuracy and throughput. In addition the delivery of targets to large facilities such as the Orion laser is presented with focus on gas-filled targets ${ }^{[9]}$.

\section{Liquid targets}

An emerging field of liquid targets for use on beamlines such as SACLA is presented ${ }^{[10]}$. Different sample injectors are presented and characterized as a route to achieve either columns, droplets or filaments as targets.

\section{High repetition rate delivery}

A thorough review paper as part of the EUCALL project is presented to determine the current state of the art in target delivery for high repetition rate sources ${ }^{[12]}$, such as XFEL. Reviews of high repetition rate developments for ICF are presented $^{[5]}$, especially at cryogenic temperatures, and also papers on the target delivery mechanisms for ion acceleration experiments are described ${ }^{[11]}$.

\section{Fundamental target fabrication}

A large number of papers cover fundamental techniques in target manufacture including fill-tube design ${ }^{[2]}$, ultrathin foils of plastic ${ }^{[13]}$, graphene ${ }^{[16]}$ and microstructures on silicon for ion sources ${ }^{[14]}$. Other research published includes beryllium-based ablator materials ${ }^{[4]}$, low density aerogels, foams, and assemblies (using low density materials) for radiation transport experiments ${ }^{[15]}$.

\section{Low density materials}

A number of papers on low density materials include the manufacture of metal doped foams ${ }^{[15]}$, including the synthesis of Fe-based aerogels using materials that are sustainable and REACH compliant ${ }^{[17]}$.

\section{Metrology techniques}

Papers on metrology cover a wide range of disciplines within target fabrication, detailing the measurement of adhesive wicking into foams ${ }^{[18]}$, the mechanical properties of ultra-thin polymer films ${ }^{[19]}$ and the surface characterization of ICF capsules ${ }^{[7]}$.

\section{Special issue content}

1. Development of target fabrication for laser-driven inertial confinement fusion at research center of laser fusion $^{[1]}$

2. Permeation fill-tube design for inertial confinement fusion target capsules ${ }^{[2]}$

3. Fluid sample injectors for X-ray free electron laser at SACLA $^{[10]}$

4. Efficient offline production of freestanding thin plastic foils for laser-driven ion sources ${ }^{[13]}$

5. A new spatial angle assembly method of the ICF target $^{[3]}$

6. An investigation progress toward Be-based ablator materials for the inertial confinement fusion ${ }^{[4]}$

7. Review on high repetition rate and mass production of the cryogenic targets for laser $\operatorname{IFE}^{[5]}$

8. An automated, $0.5 \mathrm{~Hz}$ nano-foil target positioning system for intense laser plasma experiments ${ }^{[11]}$

9. Laser-induced microstructures on silicon for laserdriven acceleration experiments ${ }^{[14]}$

10. Developing targets for radiation transport experiments at the Omega laser facility ${ }^{[15]}$

11. Targets for high repetition rate laser facilities: needs, challenges and perspectives ${ }^{[12]}$

12. Large-area suspended graphene as a laser target to produce an energetic ion beam ${ }^{[16]}$

13. Importance of limiting hohlraum leaks at cryogenic temperatures on NIF targets ${ }^{[6]}$

14. Surface characterization of ICF capsule by AFMbased profilometer ${ }^{[7]}$

15. Design and fabrication of gas cell targets for laboratory astrophysics experiments on the Orion highpower laser facility ${ }^{[9]}$

16. REACH compliant epoxides used in the synthesis of $\mathrm{Fe}$ (III)-based aerogel monoliths for target fabrication $^{[17]}$

17. Assembly and metrology of NIF target subassemblies using robotic systems ${ }^{[8]}$

18. Modeling the mechanical properties of ultra-thin polymer films ${ }^{[19]}$

19. X-ray computed tomography of adhesive wicking into carbon foam ${ }^{[18]}$ 


\section{Abstracts}

1. Development of target fabrication for laser-driven inertial confinement fusion at research center of laser fusion

Tao Wang ${ }^{(\mathrm{a} 1)}$, Kai $\mathrm{Du}^{(\mathrm{a} 1)}$, Zhibing $\mathrm{He}^{(\mathrm{a} 1)}$, and Xiaoshan $\mathrm{He}^{(\mathrm{a} 1)}$

(a1) Research Center of Laser Fusion, China Academy of Engineering Physics, Mianyang 621900, China

High Power Laser Science and Engineering Vol 5 e5 (2017)

\section{Abstract}

As the basic conditions for laser inertial confinement fusion (ICF) research, the targets are required to be well specified and elaborately fabricated. Because of the characteristics of the targets, the research and fabrication process is a systematically tough task, which needs fundamental and deep insights into film deposition, mechanical machining, precise measurement and assembly, etc. As a result, knowledge of material science, physics, mechanical as well as electronics is a necessity for target researchers. In this paper, we give introductions to the state of art on target fabrication for ICF research at Research Center of Laser Fusion (RCLF) in China.

2. Permeation fill-tube design for inertial confinement fusion target capsules

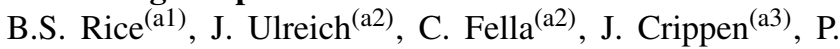
Fitzsimmons $^{(\mathrm{a} 3)}$, and A. Nikroo ${ }^{(\mathrm{a} 4)}$

(a1) Rochester Institute of Technology, 78 Lomb Memorial Drive, Rochester, NY 14623-5604, USA

(a2) Laboratory for Laser Energetics, University of Rochester, 250 East River Road, Rochester, NY 14623-1299, USA

(a3) General Atomics, San Diego, CA 92186, USA

(a4) Lawrence Livermore National Laboratory, Livermore, CA 94550, USA

\section{High Power Laser Science and Engineering Vol 5 e6 (2017)}

Abstract

A unique approach for permeation filling of nonpermeable inertial confinement fusion target capsules with deuteriumtritium (DT) is presented. This process uses a permeable capsule coupled into the final target capsule with a 0.03 mm-diameter fill tube. Leak free permeation filling of glowdischarge polymerization (GDP) targets using this method have been successfully demonstrated, as well as ice layering of the target, yielding an inner ice surface roughness of 1$\mu \mathrm{m}$ rms (root mean square). Finally, the measured DT icethickness profile for this experiment was used to validate a thermal model's prediction of the same thickness profile.

\section{Fluid sample injectors for X-ray free electron laser} at SACLA

Kensuke Tono ${ }^{(\mathrm{a} 1)}$ (a2)

(a1) Japan Synchrotron Radiation Research Institute, 1-1-1

Kouto, Sayo, Hyogo 679-5198, Japan (a2) RIKEN SPring-8 Center, 1-1-1 Kouto, Sayo, Hyogo 6795148, Japan

High Power Laser Science and Engineering Vol 5 e7 (2017)

Abstract

This paper provides a review on sample injectors which are provided at SPring-8 Angstrom Compact free electron LAser (SACLA) for conducting serial measurement in a 'diffract-before-destroy' scheme using an X-ray free electron laser (XFEL). Versatile experimental platforms at SACLA are able to accept various types of injectors, among which liquid-jet, droplet and viscous carrier injectors are frequently utilized. These injectors produce different forms of fluid targets such as a liquid filament with a diameter in the order of micrometer, micro-droplet synchronized to XFEL pulses, and slowly flowing column of highly viscous fluid with a rate below $\mu \mathrm{L} \cdot \min ^{-1}$. Characteristics and applications of the injectors are described.

\section{Efficient offline production of freestanding thin plastic foils for laser-driven ion sources}

Sebastian Seuferling ${ }^{(\mathrm{a} 1)}$, Matthias Alexander Otto Haug ${ }^{(\mathrm{a} 1)}$, Peter Hilz ${ }^{(\mathrm{a} 1)}$, Daniel Haffa ${ }^{(\mathrm{a} 1)}$, Christian Kreuzer ${ }^{(\mathrm{a} 1)}$, and Jörg Schreiber ${ }^{(\mathrm{al})}$

(a1) Fakultät für Physik, Ludwig-Maximilians-Universität München, D-85748 Garching, Germany

High Power Laser Science and Engineering Vol 5 e8 (2017)

\section{Abstract}

Modern chirped pulse amplification laser systems with continuously improving controllability and increasing power are about to reach intensities of up to $10^{22} \mathrm{~W} \cdot \mathrm{cm}^{-2}$ and have proven their potential to accelerate ions out of plasma to several tens percent of the speed of light. For enabling application, one important step is to increase the repetition rate at which ion bunches are at the disposal. In particular, techniques used so far for thin foil target production can require several days of preparing reasonable amounts for a single campaign. In this paper we describe the reasonably droplet method which we have tested and improved so that the emerging foils with thicknesses of a few nanometres up to micrometre can be used as targets for laser ion acceleration. Their quality and performance can compete with so far employed techniques thereby enabling the production of hundreds of targets per day.

5. A new spatial angle assembly method of the ICF target Wenrong $\mathrm{Wu}^{(\mathrm{a} 1)(\mathrm{a} 2)}$, Lie $\mathrm{Bi}^{(\mathrm{a} 1)(\mathrm{a} 2)}$, Kai $\mathrm{Du}^{(\mathrm{a} 1)(\mathrm{a} 2)}$, Juan Zhang $^{(\mathrm{a} 1)(\mathrm{a} 2)}$, Honggang Yang ${ }^{(\mathrm{a} 1)}$, and Honglian Wang ${ }^{(\mathrm{a} 1)}$

(a1) Research Center of Laser Fusion, CAEP, Mianyang 621900, China

(a2) Laboratory of Precision Manufacturing Technology, CAEP, China 
High Power Laser Science and Engineering Vol 5 e9 (2017)

\section{Abstract}

The designs of inertial confinement fusion (ICF) targets, which field on ShenGuang III, are becoming more complex and more stringent in terms of assembly precision. A key specification of these targets is the spatial angle alignment accuracy. To meet these needs, we present a new spatial angle assembly method, using target part's 3D model-based dual orthogonal camera vision, which is better suited for the flexible automation of target assembly processes. The two-hands structure micromanipulate system and dual orthogonal structure visual feedback system were investigated by considering the kinematics, spatial angle measuring, and motion control in an integrated way. In this paper, we discuss the measurement accuracy of spatial angle assembly method, which compared the real-time image acquisition with the redrawing $2 \mathrm{D}$ projection. The result shows that the assembly method proposed is very effective and meets the requirements of angle assembly accuracy, which is less than $1^{\circ}$. Also, this work is expected to contribute greatly to the advancement of other target microassembly equipments.

6. An investigation progress toward Be-based ablator materials for the inertial confinement fusion

Bingchi Luo (a1) (a2), Jiqiang Zhang ${ }^{(\mathrm{a} 1)}$, Yudan $\mathrm{He}^{(\mathrm{a} 1)}$, Long Chen ${ }^{(\mathrm{a} 1)}$ Jiangshan Luo ${ }^{(\mathrm{a} 1)(\mathrm{a} 2)}$, Kai Li ${ }^{(\mathrm{a} 1)(\mathrm{a} 2)}$, and Weidong $\mathrm{Wu}^{(\mathrm{a} 1)(\mathrm{a} 2)}$

(a1) Research Center of Laser Fusion, China Academy of Engineering Physics, Mianyang 621900, China

(a2) Science and Technology on Plasma Physics Laboratory, Mianyang 621900, China

High Power Laser Science and Engineering Vol 5 e10 (2017)

\section{Abstract}

The Be-based materials with many particular properties lead to an important research subject. The investigation progresses in the fabrication technologies are introduced here, including main three kinds of Be-based materials, such as $\mathrm{Be}-\mathrm{Cu}$ capsule, $\mathrm{Be}_{2} \mathrm{C}$ ablator and high-purity $\mathrm{Be}$ material. Compared with the pioneer workgroup on Be-based materials, the differences in $\mathrm{Be}-\mathrm{Cu}$ target fabrication were described, and a grain refinement technique by an active hydrogen reaction for $\mathrm{Be}$ coating was proposed uniquely. $\mathrm{Be}_{2} \mathrm{C}$ coatings were first prepared by the $\mathrm{DC}$ reactive magnetron sputtering with a high deposition rate $\left(\sim 300 \mathrm{~nm} \cdot \mathrm{h}^{-1}\right)$. Pure polycrystalline $\mathrm{Be}_{2} \mathrm{C}$ films with uniform microstructures, smooth surface, high density $\left(\sim 2.2 \mathrm{~g} \cdot \mathrm{cm}^{-3}\right)$ and good optical transparency were fabricated. In addition, the highpurity Be materials with metal impurities in a ppm magnitude were fabricated by the pyrolysis of organometallic Be.

7. Review on high repetition rate and mass production of the cryogenic targets for laser IFE

I.V. Aleksandrova ${ }^{(\mathrm{a} 1)}$ and E.R. Koresheva ${ }^{(\mathrm{a} 1)(\mathrm{a} 2)}$

(a1) Lebedev Physical Institute, Russian Academy of Sciences, Moscow 119991, Russia (a2) National Research Nuclear University MEPhI (Moscow Engineering Physics Institute), Moscow 115409, Russia

High Power Laser Science and Engineering Vol 5 e11 (2017)

\begin{abstract}
In inertial fusion energy (IFE) research, a considerable attention has recently been focused on the issue of large target fabrication for MJ-class laser facilities. The ignition and high-gain target designs require a condensed uniform layer of hydrogen fuel on the inside of a spherical shell. In this report, we discuss the current status and further trends in the area of developing the layering techniques intended to produce ignition, and layering techniques proposed to high repetition rate and mass production of IFE targets.
\end{abstract}

8. An automated, $0.5 \mathrm{~Hz}$ nano-foil target positioning system for intense laser plasma experiments

Ying Gao (a1), Jianhui Bin (a1) (a2), Daniel Haffa (a1), Christian Kreuzer ${ }^{(a 1)}$, Jens Hartmann ${ }^{(a 1)}$, Martin Speicher (a1), Florian H. Lindner ${ }^{\text {(a1) }}$, Tobias M. Ostermayr (a1) (a2), Peter Hilz ${ }^{(a 1)}$, Thomas F. Rösch ${ }^{(a 1)}$, Sebastian Lehrack (a1), Franz Englbrecht ${ }^{(a 1)}$, Sebastian Seuferling ${ }^{(a 1)}$, Max Gilljohann $^{(\mathrm{a} 1)}{ }^{(\mathrm{a} 2)}$, Hao Ding (a1) (a2), Wenjun Ma ${ }^{(\mathrm{a} 3)}$, Katia Parodi (a1), and Jörg Schreiber ${ }^{\text {(a1) (a2) }}$

(a1) Lehrstuhl für Medizinphysik, Fakultät für Physik, LudwigMaximilians-Universität München, Am Coulombwall 1, D85748, Garching, Germany

(a2) Max-Planck-Institut für Quantenoptik, D-85748 Garching, Germany

(a3) Peking University, Beijing 100871, China

High Power Laser Science and Engineering Vol 5 e12 (2017)

Abstract
We report on a target system supporting automated posi-
tioning of nano-targets with a precision resolution of $4 \mu \mathrm{m}$
in three dimensions. It relies on a confocal distance sensor
and a microscope. The system has been commissioned
to position nanometer targets with $1 \mathrm{~Hz}$ repetition rate.
Integrating our prototype into the table-top ATLAS $300 \mathrm{TW}-$
laser system at the Laboratory for Extreme Photonics in
Garching, we demonstrate the operation of a $0.5 \mathrm{~Hz}$ laser-
driven proton source with a shot-to-shot variation of the
maximum energy about $27 \%$ for a level of confidence of
0.95 . The reason of laser shooting experiments operated
at $0.5 \mathrm{~Hz}$ rather than $1 \mathrm{~Hz}$ is because the synchronization
between the nano-foil target positioning system and the laser
trigger needs to improve.
9. Laser-induced microstructures on silicon for laser-
driven acceleration experiments
Tina Ebert (a1), Nico W. Neumann ${ }^{(\text {al) }}$, Torsten Abel ${ }^{(a 1)}$,
Gabriel Schaumann ${ }^{(\mathrm{a} 1)}$, and Markus Roth ${ }^{(\mathrm{a} 1)}$
(a1) Department of Nuclear Physics, TU Darmstadt, Germany 


\section{High Power Laser Science and Engineering Vol 5 e13 (2017)}

\begin{abstract}
Ultrashort laser pulses are used to create surface structures on thin $(25 \mu \mathrm{m})$ silicon $(\mathrm{Si})$ wafers. Scanning the wafer with a galvanometric mirror system creates large homogeneously structured areas. The variety of structure shapes that can be generated with this method is exemplified by the analysis of shape, height and distance of structures created in the ambient media air and isopropanol. A study of the correlation between structure height and remaining wafer thickness is presented. The comparatively easy manufacturing technique and the structure variety that allows for custom-tailored targets show great potential for high repetition rate ion acceleration experiments.
\end{abstract}

10. Developing targets for radiation transport experiments at the Omega laser facility

D. Capelli(a1), C.A. Charsley-Groffman ${ }^{(\mathrm{a} 1)}, \quad$ R.B.

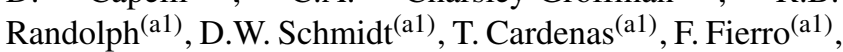
G. Rivera $^{(\mathrm{a} 1)}$, C. Hamilton ${ }^{(\mathrm{a} 1)}$, J.D. Hager ${ }^{(\mathrm{a} 1)}$, H.M. Johns ${ }^{(\mathrm{a} 1)}$, N.E. Lanier ${ }^{(\mathrm{a} 1)}$, and J.L. Kline ${ }^{(\mathrm{a} 1)}$

${ }^{(a 1)}$ Los Alamos National Laboratory - Plasma Physics, USA

\section{High Power Laser Science and Engineering Vol 5 e15 (2017)}

\section{Abstract}

Targets have been developed to measure supersonic radiation transport in aerogel foams using absorption spectroscopy. The target consists of an aerogel foam uniformly doped with either titanium or scandium inserted into an undoped aerogel foam package. This creates a localized doped foam region to provide spatial resolution for the measurement. Development and characterization of the foams is a key challenge in addition to machining and assembling the two foams so they mate without gaps. The foam package is inserted into a beryllium sleeve and mounted on a gold hohlraum. The target is mounted to a holder created using additive manufacturing and mounted on a stalk. The manufacturing of the components, along with assembly and metrology of the target are described here.

\section{Targets for high repetition rate laser facilities: needs,} challenges and perspectives

I. Prencipe ${ }^{(\mathrm{a} 1)}$, J. Fuchs ${ }^{(\mathrm{a} 2)}$, S. Pascarelli( ${ }^{(\mathrm{a} 3)}$, D.W. Schumacher $^{(\mathrm{a} 4)}$, R.B. Stephens ${ }^{(\mathrm{a} 5)}$, N.B. Alexander ${ }^{(\mathrm{a} 6)}$, R. Briggs ${ }^{(a 3)}$, M. Büscher ${ }^{(\mathrm{a} 7)(\mathrm{a} 8)}$, M.O. Cernaianu ${ }^{(\mathrm{a} 9)}$, A. Choukourov $^{(\mathrm{a} 10)(\mathrm{a} 11)}$, M. De Marco ${ }^{(\mathrm{a} 10)}, \mathrm{A} \mathrm{Erbe}^{(\mathrm{a} 12)}(\mathrm{a} 13)$, J. Fassbender ${ }^{(\mathrm{a} 12)(\mathrm{a} 13)}$, G. Fiquet ${ }^{(\mathrm{a} 14)}$, P. Fitzsimmons ${ }^{(\mathrm{a} 6)}$, C. Gheorghiu ${ }^{(a 9)}$, J. Hund ${ }^{(\mathrm{a} 15)}$, L.G. Huang ${ }^{(\mathrm{a} 1)}$, M. Harmand $^{(\mathrm{a} 14)}$, N.J. Hartley(a1) A. Irman (a1), T. Kluge ${ }^{(\mathrm{a} 1)}$,

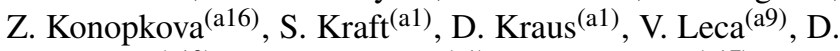
Margarone $^{(\mathrm{a} 10)}$, J. Metzkes ${ }^{(\mathrm{a} 1)}$, K. Nagai ${ }^{(\mathrm{a} 17)}$, W. Nazarov $^{(\mathrm{a} 18)}$, P. Lutoslawski ${ }^{(\mathrm{a} 10)}$, D. Papp ${ }^{(\mathrm{a} 19)}$, M. Passoni $^{(\mathrm{a} 20)(\mathrm{a} 21)}$, A. Pelka ${ }^{(\mathrm{a} 1)}$, J.P. Perin ${ }^{(\mathrm{a} 22)}$, J. Schulz ${ }^{(\mathrm{a} 16)}$,
M. Smid (a10), C. Spindloe ${ }^{(\mathrm{a} 23)(\mathrm{a} 24)}$, S. Steinke $\mathrm{e}^{(\mathrm{a} 25)}$, R.

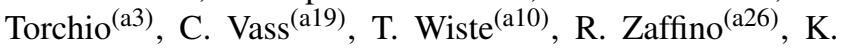
Zeil $^{(\mathrm{a} 1)}$, T. Tschentscher ${ }^{(\mathrm{a} 16)}$, U. Schramm ${ }^{(\mathrm{a} 1)(\mathrm{a} 13)}$, and T.E. Cowan ${ }^{(\mathrm{a} 1)(\mathrm{a} 13)}$

(a1) Institute of Radiation Physics, Helmholtz-Zentrum Dresden-Rossendorf, Germany

(a2) LULI - CNRS, Ecole Polytechnique, CEA: Université Paris-Saclay; UPMC Univ. Paris 06 - Sorbonne Universités - F-91128 Palaiseau cedex, France

(a3) European Synchrotron Radiation Facility, France

(a4) Ohio State University, USA

(a5) University of Pennsylvania, USA

(a6) Inertial Fusion Technologies, General Atomics, USA

(a7) Peter Grünberg Institute PGI-6, Forschungzentrum Jülich, Germany

(a8) Heinrich-Heine-University Düsseldorf, Germany

(a9) Horia Hulubei National Institute for R\&D in Physics and Nuclear Engineering (IFIN-HH) - Extreme Light Infrastructure - Nuclear Physics (ELI-NP), Romania

(a10) Institute of Physics ASCR, FZU, ELI-Beamlines project, Czech Republic

(a11) Department of Macromolecular Physics, Faculty of Mathematics and Physics, Charles University in Prague, Czech Republic

(a12) Institute of Ion Beam Physics and Materials Research, Helmholtz-Zentrum Dresden-Rossendorf, Germany (a13) Technische Universität Dresden, Germany

(a14) Institut de minéralogie, de physique des matériaux et de cosmochimie, UMR CNRS 7590, UPMC Univ. Paris 06 Sorbonne Universités, France

(a15) Schafer Corporation, USA

(a16) European XFEL, Germany

(a17) Laboratory for Chemistry and Life Science, Institute of Innovative Research (IIR), Tokyo Institute of Technology, Japan

(a18) University of St. Andrews, UK

(a19) ELI-ALPS, ELI-HU Non-Profit Ltd, Hungary

(a20) Department of Energy, Politecnico di Milano, Italy

(a21) INFN-Sezione di Milano, Italy

(a22) CEA Grenoble, INAC, Service des Basses Temperatures, France

(a23) Science and Technology Facilities Council, Rutherford Appleton Laboratory, UK

(a24) Scitech Precision Ltd, Rutherford Appleton Laboratory, UK

${ }^{(25)}$ Lawrence Berkeley National Laboratory, USA

(a26) Institute of Microelectronics of Barcelona, National Center of Microelectronic, Spanish Research Council, Spain

High Power Laser Science and Engineering Vol 5 e17 (2017)

\section{Abstract}

A number of laser facilities coming online all over the world promise the capability of high-power laser experiments with shot repetition rates between 1 and $10 \mathrm{~Hz}$. Target 
availability and technical issues related to the interaction environment could become a bottleneck for the exploitation of such facilities. In this paper, we report on target needs for three different classes of experiments: dynamic compression physics, electron transport and isochoric heating, and laserdriven particle and radiation sources. We also review some of the most challenging issues in target fabrication and high repetition rate operation. Finally, we discuss current target supply strategies and future perspectives to establish a sustainable target provision infrastructure for advanced laser facilities.

12. Large-area suspended graphene as a laser target to produce an energetic ion beam

Nur Khasanah $^{(\mathrm{a} 1)}$, Nima Bolouki ${ }^{(\mathrm{a} 1)}$, Tzu-Yao Huang ${ }^{(\mathrm{a} 1)}$, YiZhe Hong ${ }^{(\mathrm{a} 1)}$, Wen-Liang Chung ${ }^{(\mathrm{a} 1)}$, Wei-Yen Woon ${ }^{(\mathrm{a} 1)}$, Ching-Yuan $\mathrm{Su}^{(\mathrm{a} 2)}$, and Yasuhiro Kuramitsu ${ }^{(\mathrm{a} 1)}$

(a1) Department of Physics, National Central University, No. 300, Jhongda Rd., Jhongli, Taoyuan, 320 Taiwan

(a2) Graduate Institute of Energy Engineering, National Central University, No. 300, Jhongda Rd., Jhongli, Taoyuan, 320 Taiwan

\section{High Power Laser Science and Engineering Vol 5 e18} (2017)

\begin{abstract}
Proton radiography is a key diagnostics to measure and image the electric/magnetic field in laser-produced plasmas. A thin solid target is irradiated with an intense laser pulse to produce a proton beam. The accelerated proton can achieve higher energy with thinner target. In order to produce an extremely thin target, we have developed a large-area suspended graphene as a laser target for energetic ion sources. We describe the manufacturing process of the suspended graphene, and show the results of quality evaluations.
\end{abstract}

\section{Importance of limiting hohlraum leaks at cryogenic} temperatures on NIF targets

Suhas Bhandarkar ${ }^{(\mathrm{a} 1)}$, Nick Teslich ${ }^{(\mathrm{a} 1)}$, Ben Haid ${ }^{(\mathrm{a} 1)}$, and Evan Mapoles ${ }^{\text {(a1) }}$

(a1) Lawrence Livermore National Laboratory, P.O. Box 808, Livermore, CA 94550, USA

\section{High Power Laser Science and Engineering Vol 5 e19} (2017)

\section{Abstract}

Inertial confinement fusion targets are complex systems designed to allow fine control of temperature and pressure for making precise spherical ice layers of hydrogen isotopes at cryogenic temperatures. We discuss the various technical considerations for a maximum leak rate based on heat load considerations. This maximum flow rate turns out to be standard cc per second, which can be caused by an orifice less than half a micron in diameter. This makes the identification of the location and resolution of the leak a significant challenge. To illustrate this, we showcase one example of a peculiar failure mode that appeared suddenly but persisted whereby target production yield was severely lowered. Identification of the leak source and the root cause requires very careful analysis of multiple thermomechanical aspects to ensure that the end solution is indeed the right remedy and is robust.

\section{Surface characterization of ICF capsule by AFM-} based profilometer

Jie Meng ${ }^{(\mathrm{a} 1)}$, Xuesen Zhao ${ }^{(\mathrm{a} 2)}, \mathrm{Xing}_{\text {Tang }}{ }^{(\mathrm{a} 1)}$, Yihao Xia ${ }^{(\mathrm{a} 1)}$, Xiaojun $\mathrm{Ma}^{(\mathrm{a} 1)}$, and Dangzhong Gao ${ }^{(\mathrm{a} 1)}$

(a1) Research Center of Laser Fusion, CAEP, Mianyang 621000, China

(a2) P.O. Box 413, Harbin Institute of Technology, Harbin 150001, China

\section{High Power Laser Science and Engineering Vol 5 e21} (2017)

\section{Abstract}

Outside surface fluctuations of inertial confinement fusion (ICF) capsule greatly affect the implosion performance. An atomic force microscope (AFM)-based profilometer is developed to precisely characterize the capsule surface with nanometer resolution. With the standard nine surface profiles and the complete coverage data, 1D and 2D power spectra are obtained to quantitatively qualify the capsule. Capsule center fast aligning, orbit traces automatic recording, 3D capsule orientation have been studied to improve the accuracy and efficiency of the profilometer.

15. Design and fabrication of gas cell targets for laboratory astrophysics experiments on the Orion high-power laser facility

C. Spindloe ${ }^{(\mathrm{a} 1)}$, D. Wyatt $^{(\mathrm{a} 1)}$, S. Astbury ${ }^{(\mathrm{a} 1)}$, G.F. Swadling $^{(\mathrm{a} 2)(\mathrm{a} 3)}$ T. Clayson $^{(\mathrm{a} 2)}$, C. Stehlé( ${ }^{(\mathrm{a} 4)}$, J.M. Foster $^{(\mathrm{a} 5)}$,

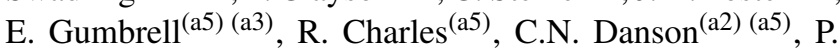
Brummitt $^{(\mathrm{a} 1)}$, and F. Suzuki-Vidal ${ }^{(\mathrm{a} 2)}$

(a1) Science and Technology Facilities Council, Harwell Science and Innovation Campus, Didcot, OX11 0QX, UK

(a2) Blackett Laboratory, Imperial College, London, SW7 2BW, UK

(a3) Current address: Lawrence Livermore National Laboratory, California 94550, USA

(a4) LERMA, Sorbonne Universités, UPMC Univ. Paris 06, Observatoire de Paris, PSL Research University, CNRS, F-75252, Paris, France

(a5) AWE plc, Aldermaston, Reading RG7 4PR, UK

High Power Laser Science and Engineering Vol 5 e22 (2017)
Abstract
This paper describes the design and fabrication of a range of 'gas cell' microtargets produced by the Target Fabrication Group in the Central Laser Facility (CLF) for 
academic access experiments on the Orion laser facility at the Atomic Weapons Establishment (AWE). The experiments were carried out by an academic consortium led by Imperial College London. The underlying target methodology was an evolution of a range of targets used for experiments on radiative shocks and involved the fabrication of a precision machined cell containing a number of apertures for interaction foils or diagnostic windows. The interior of the cell was gas-filled before laser irradiation. This paper details the assembly processes, thin film requirements and micro-machining processes needed to produce the targets. Also described is the implementation of a gas-fill system to produce targets that are filled to a pressure of $0.1-1$ bar. The paper discusses the challenges that are posed by such a target.

16. REACH compliant epoxides used in the synthesis of Fe(III)-based aerogel monoliths for target fabrication

Alberto Valls Arrufat ${ }^{(a 1)}$, Magdalena Budziszewska( ${ }^{(a 1)}$, Clement Lopez ${ }^{(\mathrm{a} 1)}$, Aymeric Nguyen ${ }^{(\mathrm{a} 1)}$, Jakub Sitek ${ }^{(\mathrm{a} 1)}$, Paul Jones ${ }^{(\mathrm{a} 1)}$, Chris Shaw ${ }^{(\mathrm{a} 1)}$, Ian Hayes ${ }^{(\mathrm{a} 2)}$, Gareth Cairns $^{(\mathrm{a} 2)}$, and Glenn Leighton ${ }^{(\mathrm{a} 1)}$

(a1) Surface Engineering and Nanotechnology Institute, School of Aerospace, Transport and Manufacturing, Cranfield University, Milton Keynes, Bedfordshire, MK43 0AL, UK

(a2) Target Fabrication Group, AWE, Aldermaston, Reading, Berkshire, RG7 4PR, UK

\section{High Power Laser Science and Engineering Vol 5 e24 (2017)}

\section{Abstract}

Aerogel materials manufactured from metal oxides have been used as components in numerous high-energy density physics targets. These aerogels have been identified to be used as a future target material in the AWE fielded campaigns at the US National Ignition Facility. A wide variety of metal oxide aerogels are required for future campaigns and therefore a versatile manufacturing route is sought; as such, an epoxide-assisted sol-gel route was investigated. Under the European Union Registration, Evaluation, Authorization and Restriction of Chemicals legislation, the most commonly used epoxide, propylene oxide, is recognized as a substance of very high concern (SVHC). This work sought to investigate suitable alternative epoxides for use in target manufacture. The outcome was the identification of synthesis routes for stable metal oxide aerogel monoliths using epoxides not subject to the above restrictions.

\section{Assembly and metrology of NIF target subassemblies using robotic systems}

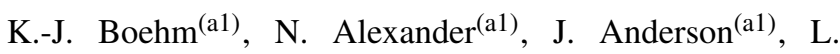
Carlson $^{(\mathrm{a} 1)}$, and M. Farrell ${ }^{(\mathrm{a} 1)}$

(a1) General Atomics, P.O. Box 85608, San Diego, CA 92186-5608, USA
High Power Laser Science and Engineering Vol 5 e25 (2017)

\section{Abstract}

With European Laser Facilities such as the Extreme Light Infrastructure (ELI) and the Helmholtz International Beamline for Extreme Fields (HIBEF) scheduled to come online within the next couple of years, General Atomics, as a major supplier of targets and target components for the High Energy Density Physics community in the United States, is gearing up to meet their demand for large numbers of low cost targets. Using the production of a subassembly for the National Ignition Facility's fusion targets as an example, we demonstrate that through automation of assembly tasks, the design of targets and their experimental setup can be fairly complex while keeping the assembly time and cost as a minimum. A six-axis Mitsubishi robot is used in combination with vision feedback and a forcetorque sensor to assemble target subassemblies of different scales and designs with minimal change of tooling, allowing for design flexibility and short assembly setup times. Implementing automated measurement routines on a Nikon NEXIV microscope further reduces the effort required for target metrology, while electronic data collection and transfer complete a streamlined target production operation that can be adapted to a large variety of target designs.

18. Modeling the mechanical properties of ultra-thin polymer films

Francisco Espinosa-Loza ${ }^{(\mathrm{a} 1)}$, Michael Stadermann ${ }^{(\mathrm{a} 1)}$, Chantel Aracne-Ruddle ${ }^{(a 1)}$, Rebecca Casey ${ }^{(a 1)}$, Philip Miller $^{(\mathrm{a} 1)}$, and Russel Whitesides ${ }^{(\mathrm{a} 1)}$

(a1) Lawrence Livermore National Laboratory, 7000 East Avenue, L-471, Livermore, CA 94550, USA

\section{High Power Laser Science and Engineering Vol 5 e27} (2017)

\section{Abstract}

A modeling method to extract the mechanical properties of ultra-thin films (10-100 nm thick) from experimental data generated by indentation of freestanding circular films using a spherical indenter is presented. The relationship between the mechanical properties of the film and experimental parameters including load, and deflection are discussed in the context of a constitutive material model, test variables, and analytical approaches. Elastic and plastic regimes are identified by comparison of finite element simulation and experimental data.

\section{X-ray computed tomography of adhesive wicking into carbon foam}

Sav Chima ${ }^{(a 1)}$

(a1) AWE plc, Aldermaston, UK 


\section{High Power Laser Science and Engineering Vol 5 e28 (2017)}

\begin{abstract}
Laser target components consist of multicomponent porous and nonporous materials that are adhesively bonded together. In order to assess the extent and quantity of adhesive wicking into porous foam, micro X-ray computed tomography (CT) and image processing software have been utilized. Two different laser target configurations have been assessed in situ and volume rendered images of the distribution and quantities of adhesive have been determined for each.
\end{abstract}

\section{References}

1. T. Wang, K. Du, Z. He, and X. He, High Power Laser Sci. Eng. 5, e5 (2017).

2. B. S. Rice, J. Ulreich, C. Fella, J. Crippen, P. Fitzsimmons, and A. Nikroo, High Power Laser Sci. Eng. 5, e6 (2017).

3. W. Wu, L. Bi, K. Du, J. Zhang, H. Yang, and H. Wang, High Power Laser Sci. Eng. 5, e9 (2017).

4. B. Luo, J. Zhang, Y. He, L. Chen, J. Luo, K. Li, and W. Wu, High Power Laser Sci. Eng. 5, e10 (2017).

5. I. V. Aleksandrova and E. R. Koresheva, High Power Laser Sci. Eng. 5, e11 (2017).

6. S. Bhandarkar, N. Teslich, B. Haid, and E. Mapoles, High Power Laser Sci. Eng. 5, e19 (2017).

7. J. Meng, X. Zhao, X. Tang, Y. Xia, X. Ma, and D. Gao, High Power Laser Sci. Eng. 5, e21 (2017).

8. K.-J. Boehm, N. Alexander, J. Anderson, L. Carlson, and M. Farrell, High Power Laser Sci. Eng. 5, e25 (2017).

9. C. Spindloe, D. Wyatt, S. Astbury, G. F. Swadling, T. Clayson, C. Stehlé, J. M. Foster, E. Gumbrell, R. Charles, C. N. Danson,
P. Brummitt, and F. Suzuki-Vidal, High Power Laser Sci. Eng. 5, e22 (2017)

10. K. Tono, High Power Laser Sci. Eng. 5, e7 (2017).

11. Y. Gao, J. Bin, D. Haffa, C. Kreuzer, J. Hartmann, M. Speicher, F. H. Lindner, T. M. Ostermayr, P. Hilz, T. F. Rösch, S. Lehrack, and F. Englbrecht, High Power Laser Sci. Eng. 5, e12 (2017).

12. I. Prencipe, J. Fuchs, S. Pascarelli, D. W. Schumacher, R. B. Stephens, N. B. Alexander, R. Briggs, M. Büscher, M. O. Cernaianu, A. Choukourov, M. De Marco, A. Erbe, J. Fassbender, G. Fiquet, P. Fitzsimmons, C. Gheorghiu, J. Hund, L. G. Huang, M. Harmand, N. J. Hartley, A. Irman, T. Kluge, Z. Konopkova, S. Kraft, D. Kraus, V. Leca, D. Margarone, J. Metzkes, K. Nagai, W. Nazarov, P. Lutoslawski, D. Papp, M. Passoni, A. Pelka, J. P. Perin, J. Schulz, M. Smid, C. Spindloe, S. Steinke, R. Torchio, C. Vass, T. Wiste, R. Zaffino, K. Zeil, T. Tschentscher, U. Schramm, and T. E. Cowan, High Power Laser Sci. Eng. 5, e17 (2017).

13. S. Seuferling, M. Alexander, O. Haug, P. Hilz, D. Haffa, C. Kreuzer, and J. Schreiber, High Power Laser Sci. Eng. 5, e8 (2017).

14. T. Ebert, N. W. Neumann, T. Abel, G. Schaumann, and M. Roth, High Power Laser Sci. Eng. 5, e13 (2017).

15. D. Capelli, C. A. Charsley-Groffman, R. B. Randolph, D. W. Schmidt, T. Cardenas, F. Fierro, G. Rivera, C. Hamilton, J. D. Hager, H. M. Johns, N. E. Lanier, and J. L. Kline, High Power Laser Sci. Eng. 5, e15 (2017).

16. N. Khasanah, N. Bolouki, T.-Y. Huang, Y.-Z. Hong, W.-L. Chung, W.-Y. Woon, C.-Y. Su, and Y. Kuramitsu, High Power Laser Sci. Eng. 5, e18 (2017).

17. A. V. Arrufat, M. Budziszewska, C. Lopez, A. Nguyen, J. Sitek, P. Jones, C. Shaw, I. Hayes, G. Cairns, and G. Leighton, High Power Laser Sci. Eng. 5, e24 (2017).

18. S. Chima, High Power Laser Sci. Eng. 5, e28 (2017).

19. F. Espinosa-Loza, M. Stadermann, C. Aracne-Ruddle, R. Casey, P. Miller, and R. Whitesides, High Power Laser Sci. Eng. 5, e27 (2017). 